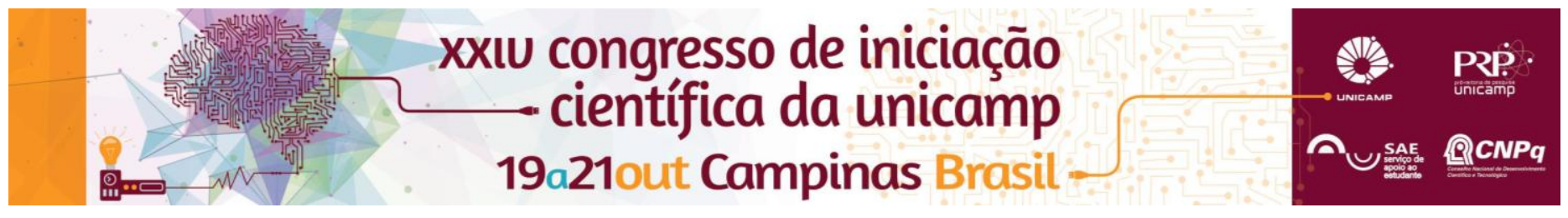

\title{
Chemical cross-linked nanocellulose-based gels: preparation and characterization
}

\author{
Victor Z. e Resende*, Liliane C. Battirola, Maria do Carmo Gonçalves.
}

\begin{abstract}
Chemical modification of cellulose nanocrystals (CNC) is an interesting alternative to prepare chemical cross-linked nanocellulose-based gels. In this work, chemically cross-linked CNC gels were prepared by the reaction of aldehydefunctionalized CNC and two different diamines: ethylenediamine (ED) and 4,4'-(1,3-phenylene dioxy)dianiline (PDD). Chemical modifications were verified by FTIR and POM. The formation of a solid gel was achieved when PDD was used as the cross-linking agent.
\end{abstract}

Key words: Cellulose nanocrystal, chemical cross-linking, gel.

\section{Introduction}

Cellulose nanocrystals (CNC) present high mechanical properties, renewability, and high surface reactivity, which allow the CNC applicability in a variety of materials. CNCbased gels, in particular, are promising materials for use when high absorbent, strength and stiffness properties are required, apart from their applicability as biomaterials. However, the challenge of making CNC gels is its inability to form effective entanglements between individual rigid particles. An alternative to overcome this obstacle is to introduce different chemical functionalities through the hydroxyl group reaction on the CNC surface ${ }^{1}$. Therefore, the aim of this work is to prepare CNC cross-linking networks.

\section{Results and Discussion}

Cotton fibers were hydrolyzed in 65 wt\% $\mathrm{H}_{2} \mathrm{SO}_{4}$ at $55{ }^{\circ} \mathrm{C}$ for $35 \mathrm{~min}$ under stirring. Cold water was added to interrupt the reaction and the excess acid was removed by repeated centrifugation cycles. CNC suspension was then dialyzed until neutral $\mathrm{pH}$ was reached then lyophilized to obtain CNC powder. CNC was submitted to two different reactions. In Reaction 1, periodate oxidation $\left(\mathrm{NalO}_{4}\right)$ was carried out in a lightproof flask; $\mathrm{NalO}_{4}$ was dissolved in CNC suspension at $38^{\circ} \mathrm{C}$ for $24 \mathrm{~h}$ under stirring, ethylene glycol was added to react for $1 \mathrm{~h}$ under stirring and then dialyzed. In Reaction 2, both ethylenediamine (ED) and 4,4'-(1,3-phenylene dioxy)dianiline (PDD) cross-linking agents were added to the reaction 1 product. Therefore, an appropriate amount of diamine was added to the oxidized CNC suspension (CNC-C=O, Reaction 1 product), and the $\mathrm{pH}$ was adjusted to reach a 4-5 value. The reaction was carried out at $45{ }^{\circ} \mathrm{C}$ for $4 \mathrm{~h}$ under stirring. Finally, the functionalized CNC (CNC-C=N-ED or CNC-C=N-PDD, Reaction 2 product) was dialyzed until reaching a $\mathrm{pH}$ of 7 , and the remaining diamine compound was completely removed. Surface fuctionalization reactions were confirmed by FTIR (Fourier Transform Infrared Spectroscopy) and POM (Polarized Light Microscopy). In Image 1, the FTIR spectrum of the Reaction 1 product showed the $\mathrm{C}=\mathrm{O}$ stretching at $1725 \mathrm{~cm}^{-1}$ and the absence of this peak after imine bond formation for both Reaction 2 products. POM images showed that the CNC hydrophilic character was changed as a consequence of the surface functionalization. The results showed that the reaction carried out with ED promoted the CNC surface functionalization, but no gel formation (Image 2a). On the other hand, a gel was obtained when PDD was used,

DOI: 10.19146/pibic-2016-51712 which suggests the formation of chemical cross-linking between $\mathrm{CNC}-\mathrm{C}=\mathrm{O}$ and $\mathrm{PDD}$, as observed in Image $2 \mathrm{~b}$. Images $2 \mathrm{c}$ and $2 \mathrm{~d}$ show the electron micrographs of the dried materials, indicating two different morphologies: spherical micelles and regular porous structure for CNC$\mathrm{C}=\mathrm{N}-\mathrm{ED}$ and $\mathrm{CNC}-\mathrm{C}=\mathrm{N}-\mathrm{PDD}$, respectively.

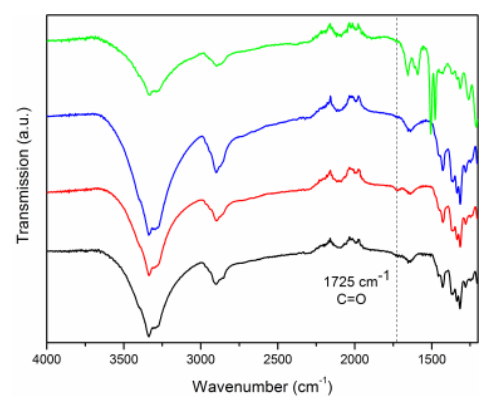

Image 1. FTIR spectrum of CNC (-), CNC-C=O (-), CNC$\mathrm{C}=\mathrm{N}-\mathrm{ED}(-)$ and $\mathrm{CNC}-\mathrm{C}=\mathrm{N}-\mathrm{PDD}(-)$.
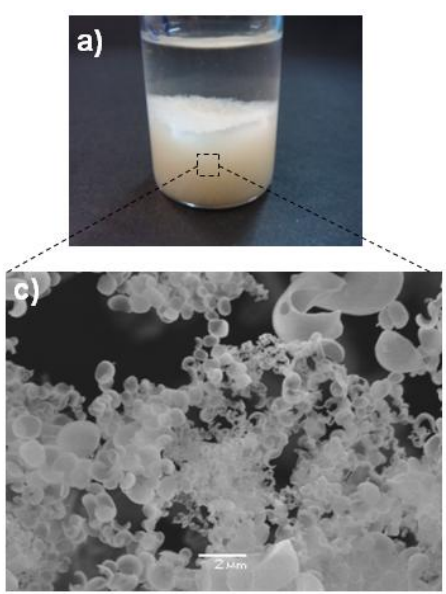
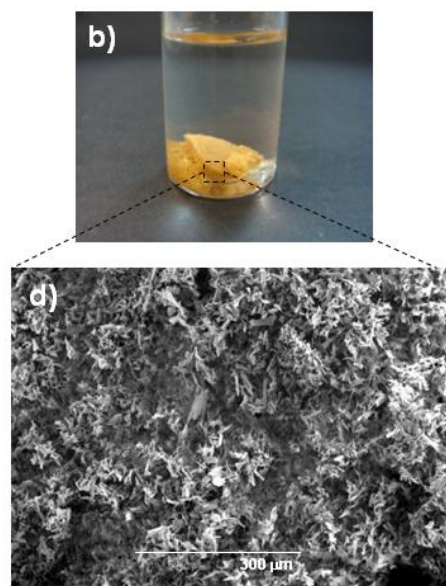

Image 2. Photographs of (a) CNC-C=N-ED and (b) CNC$\mathrm{C}=\mathrm{N}-\mathrm{PDD}$ gel and FESEM micrographs of (c) $\mathrm{CNC}-\mathrm{C}=\mathrm{N}$ ED and (d) CNC-C=N-PDD gel.

\section{Conclusions}

CNC surface functionalizations using both ED and PDD were confirmed by FTIR and POM. The results indicate that the formation of chemical cross-linking was achieved when PDD was used as a cross-linking agent.

\section{Acknowledgement}

$\mathrm{PIBIC/CNPq}$, FAPESP and CAPES. 